\title{
Strange particle production at RHIC in the Dual Parton Model
}

\author{
A. Capella ${ }^{\mathrm{a})}$, C.A. Salgado ${ }^{\mathrm{b})}$, D. Sousa ${ }^{\mathrm{c})}$ \\ a) Laboratoire de Physique Théoriquef \\ Université de Paris XI, Bâtiment 210, F-91405 Orsay Cedex, France \\ b) Theory Division, CERN, CH-1211 Geneva 23, Switzerland \\ c) ECT* $^{*}$, Trento, Italy
}

\begin{abstract}
We compute the mid-rapidity densities of pions, kaons, baryons and antibaryons in $A u-A u$ collisions at $\sqrt{s}=130 \mathrm{GeV}$ in the Dual Parton Model supplemented with final state interaction (comovers interaction). The ratios $B / n_{\text {part }}\left(\bar{B} / n_{\text {part }}\right)$ increase between peripheral $\left(n_{\text {part }}=18\right)$ and central $\left(n_{\text {part }}=350\right)$ collisions by a factor $2.4(2.0)$ for $\Lambda$ 's, 4.8 (4.1) for $\Xi$ 's and 16.5 (13.5) for $\Omega^{\prime}$ 's. The ratio $K^{-} / \pi^{-}$increases by a factor 1.3 in the same centrality range. A comparison with available data is presented.
\end{abstract}

LPT Orsay 02-09

CERN-TH/2002-090

$\mathrm{ECT}^{*} 02-10$

February 2002

\footnotetext{
${ }^{1}$ Unité Mixte de Recherche UMR n 8627 - CNRS
} 
The enhancement of the ratio of yields of strange baryons and antibaryons per participant nucleon, observed at CERN-SPS, [1] [2], is one of the main results of the Heavy Ion CERN program. A description of these data has been given in [3] in

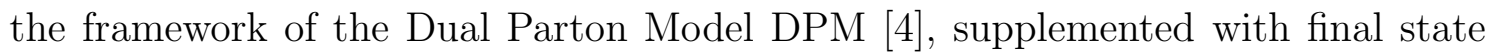
interaction. The net baryon yield is computed in the same framework taking into account the mechanism of baryon stopping, associated with baryon junction transfer in rapidity [5 9]. We use its implementation in [3], which describes the SPS data.

In the absence of nuclear shadowing, the rapidity density of a given type of hadron $h$ produced in $A A$ collisions at fixed impact parameter, is given by [4] [10]

$$
\begin{aligned}
\frac{d N^{A A \rightarrow h}}{d y}(y, b)= & n_{A}(b)\left[N_{h, \mu(b)}^{q q^{P}-q_{v}^{T}}(y)+N_{h, \mu(b)}^{q_{v}^{P}-q q^{T}}(y)+(2 k-2) N_{h, \mu(b)}^{q_{s}-\bar{q}_{s}}(y)\right] \\
& +\left(n(b)-n_{A}(b)\right) 2 k N_{h, \mu(b)}^{q_{s}-\bar{q}_{s}}(y) .
\end{aligned}
$$

Here

$$
n(b)=\sigma_{p p} A^{2} \int d^{2} s T_{A}(s) T_{B}(b-s) / \sigma_{A A}(b)
$$

is the average number of binary collisions and

$$
n_{A}(b)=A \int d^{2} s T_{A}(s)\left[1-\exp \left(-\sigma_{p p} A T_{A}(b-s)\right] / \sigma_{A A}(b),\right.
$$

is the average number of participant pairs at fixed impact parameter $b . P$ and $T$ denote projectile and target nuclei. $k$ is the average number of inelastic collisions in $p p$ and $\mu(b)=k \nu(b)$ with $\nu(b)=n(b) / n_{A}(b)$ is the average total number of collisions suffered by each nucleon. At $\sqrt{s}=130 \mathrm{GeV}$ we have $k=2$ [10].

The $N_{h, \mu(b)}(y)$ in eq. (1) are the rapidity distributions of hadron $h$ in each individual string. In DPM they are given by convolutions of momentum distribution and fragmentation functions]. The first term in (1) is the rapidity distribution in one $N N$ collision of $A A$, resulting from the superposition of $2 k$ strings, multiplied by the average number of participant pairs. Since in DPM there are two strings

\footnotetext{
${ }^{2}$ For pions, we use the same fragmentation functions given in [10]. For simplicity, the same form is used for kaons. For $p \bar{p}$ pair production we take 11] $x D_{q q}^{p}(x)=x D_{q q}^{\bar{p}}(x) \sim(1-x)^{5}$ and $x D_{q}^{p}(x)=x D_{q}^{\bar{p}}(x) \sim(1-x)^{3}$. For the other baryon species an extra $\alpha_{\rho}(0)-\alpha_{\phi}(0)=1 / 2$ is added to the power of $(1-x)$ for each strange quark in the baryon 11 .
} 
per inelastic collision, the second term, consisting of strings stretched between sea quarks and antiquarks, makes up for the total average number of strings $2 k n(b)$.

It was shown in [10] that eq. (11), supplemented with shadowing corrections, leads to values of charged multiplicities at mid-rapidities as a function of centrality in agreement with data, both at SPS and RHIC. Here we use the same shadowing corrections as in ref. [10 - leading to the lower edge of the shaded area in Fig. 4 of 10].

Net Baryon Production. Let us now consider the net baryon production $\Delta B=$ $B-\bar{B}$. In the standard version of DPM [4] (or QGSM [12]) the leading baryon results from the fragmentation of a valence diquark. This component will be called diquark preserving (DP). The stopping observed in $\mathrm{Pb} \mathrm{Pb}$ collisions at SPS has led to the introduction of a new mechanism based on the transfer in rapidity of the baryon junction [5 9]. Here we follow the formalism in [3] which describes the SPS data. In an $A A$ collision, this component, called diquark-breaking (DB), gives the following rapidity distribution of the two net baryons in a single $N N$ collision of $A A$ 3

$$
\left(\frac{d N_{D B}^{\Delta B}}{d y}(y)\right)_{\nu(b)}=C_{\nu(b)}\left[Z_{+}^{1 / 2}\left(1-Z_{+}\right)^{\nu(b)-3 / 2}+Z_{-}^{1 / 2}\left(1-Z_{-}\right)^{\nu(b)-3 / 2}\right]
$$

where $Z_{ \pm}=\exp \left( \pm y-y_{\max }\right)$ and $\nu(b)=n(b) / n_{A}(b) . C_{\nu(b)}$ is determined from the normalization to two at each $b$.

The net baryon rapidity distribution in $A A$ collisions is then given by

$$
\frac{d N^{A A \rightarrow \Delta B}}{d y}(y, b)=n_{A}(b)\left[\frac{1}{\nu(b)}\left(\frac{d N_{D P}^{\Delta B}}{d y}(y)\right)_{\nu(b)}+\frac{\nu(b)-1}{\nu(b)}\left(\frac{d N_{D B}^{\Delta B}}{d y}(y)\right)_{\nu(b)}\right] .
$$

The physical content of eq. (5) is as follows. Each nucleon interacts in average with $\nu(b)$ nucleons of the other nucleus. It has been argued in [3] that in only one of these collisions the string junction, carrying the baryon number, follows a valence diquark, which fragments according to the DP mechanism. In the $\nu(b)-1$ others, the string junction is freed from the valence diquark and net baryon production takes place according to the DB mechanism, eq. (4). In order to conserve baryon 
number, we have to divide by $\nu(b)$ and multiply by the number of participating nucleons. We obtain in this way eq. (5) $)^{\text {] }}$. This equation gives the total net baryon densityf.

In order to get the relative densities of each baryon and antibaryon species we use simple quark counting rules [3]. Denoting the strangeness suppression factor by $S / L$ (with $2 L+S=1$ ), baryons produced out of three sea quarks (which is the case for pair production) are given the relative weights

$$
I_{3}=4 L^{3}: 4 L^{3}: 12 L^{2} S: 3 L S^{2}: 3 L S^{2}: S^{3} .
$$

for $p, n, \Lambda+\Sigma, \Xi^{0}, \Xi^{-}$and $\Omega$, respectively. The various coefficients of $I_{3}$ are obtained from the power expansion of $(2 L+S)^{3}$. In order to take into account the decay of $\Sigma^{*}(1385)$ into $\Lambda \pi$, we redefine the relative rate of $\Lambda$ 's and $\Sigma$ 's using the empirical rule $\Lambda=0.6\left(\Sigma^{+}+\Sigma^{-}\right)$- keeping, of course, the total yield of $\Lambda^{\prime}$ s plus $\Sigma$ 's unchanged. In this way the normalization constants of all baryon species in pair production are determined from one of them. This constant, together with the relative normalization of $K$ and $\pi$, are determined from the data for very peripheral collisions. In the calculations we use $S=0.1(S / L=0.22)$.

For net baryon production two possibilities have been considered. The first one is that the behaviour in $Z^{1 / 2}$, eq. (41), is associated to the transfer of the string junction without valence quarks [5, 7,9]. In this case the net baryon is made out of three sea quarks and the relevant weights are given by $I_{3}$. In the second one, eq. (州) is a pre-asymptotic term associated to the transfer of the baryon junction plus one valence quark [6]. In this case the relevant weights are given by $I_{2}$, i.e. from the various terms in the expansion of $(2 L+S)^{2}$. This second possibility is favored by the dataf. Since the normalization of the total net baryon yield is determined

\footnotetext{
${ }^{3}$ In the numerical calculations we neglect the first term of (5) since the $D P$ component gives a very small contribution at $y^{*} \sim 0$ and RHIC energies - about $5 \%$ of the $D B$ one for the most central bin where its effect is maximal.

${ }^{4}$ In order to conserve strangeness locally, we have added an extra $1 / 2 K^{+}$and $1 / 2 K^{0}$ to each produced net $\Lambda$ (plus $\Sigma$ 's), an extra $K^{+}$and $K^{0}$ to each net $\Xi$ and an extra $3 / 2 K^{+}$and $3 / 2 K^{0}$ to each net $\Omega$.

${ }^{5}$ Note, however, that a non-zero value of net omegas has been observed in $h A$ collisions [13]. This requires a non-vanishing contribution proportional to $I_{3}$. However, its effect in $A A$ collisions is presumably small since, in this case, the net omegas are almost entirely due to final state
} 
from baryon number conservation, there is no extra free normalization constant. Moreover, the total net baryon yield is not affected by final state interaction.

Final State Interactions. The hadronic densities obtained above will be used as initial conditions in the gain and loss differential equations which govern final state interactions. In the conventional derivation [14] of these equations, one uses cylindrical space-time variables and assumes boost invariance. Furthermore, one assumes that the dilution in time of the densities is only due to longitudinal motion, which leads to a $\tau^{-1}$ dependence on the longitudinal proper time $\tau$. These equations can be written as [14] [3]

$$
\tau \frac{d \rho_{i}}{d \tau}=\sum_{k \ell} \sigma_{k \ell} \rho_{k} \rho_{\ell}-\sum_{k} \sigma_{i k} \rho_{i} \rho_{k}
$$

The first term in the r.h.s. of (6) describes the production (gain) of particles of type $i$ resulting from the interaction of particles $k$ and $\ell$. The second term describes the loss of particles of type $i$ due to its interaction with particles of type $k$. In eq. (6) $\rho_{i}=d N_{i} / d y d^{2} s(y, b)$ are the particles yields per unit rapidity and per unit of transverse area, at fixed impact parameter. They can be obtained from the rapidity densities (11), (5) using the geometry, i.e. the $s$-dependence of $n_{A}$ and $n$, eqs. (2), (3). The procedure is explained in detail in [15]. $\sigma_{k \ell}$ are the corresponding cross-sections averaged over the momentum distribution of the colliding particles.

Equations (6) have to be integrated from initial time $\tau_{0}$ to freeze-out time $\tau_{f}$. They are invariant under the change $\tau \rightarrow c \tau$ and, thus, the result depends only on the ratio $\tau_{f} / \tau_{0}$. We use the inverse proportionality between proper time and densities and put $\tau_{f} / \tau_{0}=\left(d N / d y d^{2} s(y, b)\right) / \rho_{f}$. Here the numerator is given by the DPM particles densities. We take $\rho_{f}=\left[3 / \pi R_{p}^{2}\right]\left(d N^{-} / d y\right)_{y^{*} \sim 0}=2 \mathrm{fm}^{-2}$, which corresponds to the charged density per unit rapidity in a $p p$ collision at $\sqrt{s}=$ $130 \mathrm{GeV}$. This density is about $70 \%$ larger [10] than at SPS energies. Since the corresponding increase in the $A A$ density is comparable, the average duration time of the interaction will be approximately the same at CERN SPS and RHIC - about 5 to $7 \mathrm{fm}$.

interactions. 
Next, we specify the channels that have been taken into account in our calculations. They are

$$
\pi N \rightleftarrows K \Lambda(\Sigma), \quad \pi \Lambda(\Sigma) \rightleftarrows K \Xi, \quad \pi \Xi \rightleftarrows K \Omega
$$

We have also taken into account the strangeness exchange reactions

$$
\pi \Lambda(\Sigma) \rightleftarrows K N, \quad \pi \Xi \rightleftarrows K \Lambda(\Sigma), \quad \pi \Omega \rightleftarrows K \Xi
$$

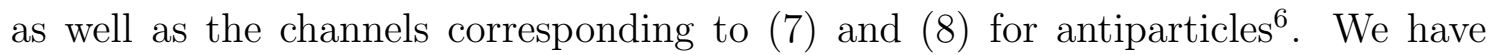
taken $\sigma_{i k}=\sigma=0.2 \mathrm{mb}$, i.e. a single value for all reactions in (7) and (8) - the same value used in ref. 3] to describe the CERN SPS data.

Numerical Results. All our results refer to mid-rapidities. The calculations have been performed in the interval $-0.35<y^{*}<0.35$. In Fig. 1a-1d we show the rapidity densities of $B, \bar{B}$ and $B-\bar{B}\left[\right.$ versus $h^{-}=d N^{-} / d \eta=(1 / 1.17) d N / d y$ and compare them with available data [17 19]. We see that, in first approximation, $p$, $\bar{p}, \Lambda$ and $\bar{\Lambda}$ scale with $h^{-}$. Quantitatively, there is a slight decrease with centrality of $p / h^{-}$and $\bar{p} / h^{-}$ratios, a slight increase of $\Lambda / h^{-}$and $\bar{\Lambda} / h^{-}$and a much larger increase for $\Xi(\bar{\Xi}) / h^{-}$and $\Omega(\bar{\Omega}) / h^{-}$. In Fig. 2a and $2 \mathrm{~b}$ we plot the yields of $B$ and $\bar{B}$ per participant normalized to the same ratio for peripheral collisions versus $n_{\text {part }}$. The enhancement of $B$ and $\bar{B}$ increases with the number of strange quarks in the baryon. This increase is comparable to the one found at SPS between pA and central $\mathrm{PbPb}$ collisions - somewhat larger for antibaryons. The ratio $K^{-} / \pi^{-}$ increases by $30 \%$ in the same centrality range, between 1.1 and 1.4 in agreement with present data [17]. The ratios $\bar{B} / B$ have a mild decrease with centrality of

\footnotetext{
${ }^{6}$ To be precise, of all possible charge combinations in reactions (7), we have only kept those involving the annihilation of a light $q-\bar{q}$ pair and production of an $s-\bar{s}$ in the $s$-channel. The other reactions, involving three quarks in the $t$-channel intermediate state, have substantially smaller cross-sections and have been neglected. All channels involving $\pi^{0}$ have been taken with crosssection $\sigma / 2$ since only one of the $u \bar{u}$ and $d \bar{d}$ components of $\pi^{0}$ can participate to a given charge combination. For details see the second paper of [3].

${ }^{7} \mathrm{~A}$ Monte Carlo calculation in a similar framework with string fusion can be found in [16]. A net proton rapidity density of about 10 for central $A u A u$ collisions at mid-rapidities at RHIC was first predicted in [8] using a stopping mechanism similar to the one considered here.
} 
about $15 \%$ for all baryon species - which is also seen in the data 21. Our values for $N^{c h} / N_{\max }^{c h}=1 / 2$ are $: \bar{p} / p=0.69, \bar{\Lambda} / \Lambda=0.72, \Xi / \Xi=0.79, \Omega / \bar{\Omega}=0.83$, to be compared with the measured values [18] :

$$
\bar{p} / p=0.63 \pm 0.02 \pm 0.06 \quad, \quad \bar{\Lambda} / \Lambda=0.73 \pm 0.03 \quad, \quad \Xi / \Xi=0.83 \pm 0.03 \pm 0.05
$$

The ratio $K^{+} / K^{-}=1.1$ and has a mild increase with centrality, a feature also seen in the data.

As explained above only one parameter has been adjusted in order to determine the absolute yields of baryons and antibaryons. Since both the PHENIX [17 and the STAR data [19] are not corrected for feed-down from weak decays, this free parameter has to be re-determined after these corrections are known. It will then be possible to compare its value with the one obtained from other sets of data, in particular $p p$. Likewise, the corrected yields of net protons will determine the exact amount of stopping and should allow to decide whether or not there is "anomalous" stopping in $A A$, i.e. an excess as compared to an extrapolation from $p p$ and $p A$. At present there is no clear indication of anomalous stopping.

After completion of this work, the PHENIX collaboration [24] has published the yields of $p$ and $\bar{p}$ for the $5 \%$ most central events corrected for feed-down. The corrections are of $30 \%$. The systematic error is $20 \%$.Also, C.Roy (STAR collaboration) communicated to us a recent STAR preliminary result on the mid-rapidity densities of $\Xi^{-}$and $\Xi^{+}$for the $14 \%$ most central events. These values are 60 to $70 \%$ higher than our results. Their systematic error is $20 \%$.

Physical interpretation. Before final state interactions, all ratios $K / h^{-}, B / h^{-}$ and $\bar{B} / h^{-}$decrease slightly with increasing centrality. This effect is rather marginal at RHIC energies and mid-rapidities.

The final state interactions (7), (8) lead to a gain of strange particle yields. The reason for this is the following. In the first direct reaction (7) we have $\rho_{\pi}>\rho_{K}$, $\rho_{N}>\rho_{\Lambda}, \rho_{\pi} \rho_{N} \gg \rho_{K} \rho_{\Lambda}$. The same is true for all direct reaction (7). In view of that, the effect of the inverse reactions (7) is small. On the contrary, in all reactions

\footnotetext{
${ }^{8}$ In ref. [3], the relative weights of net baryons were given by the factors $0.5\left(I_{2}+I_{3}\right)$ - instead of $I_{2}$. In this case the values of the ratios are $\bar{p} / p=0.70, \bar{\Lambda} / \Lambda=0.71, \Xi / \Xi=0.76$ and $\bar{\Omega} / \Omega=0.78$. Their increase with the number of strange quarks in the baryon is smaller.
} 
(8), the product of densities in the initial and final state are comparable and the direct and inverse reactions tend to compensate with each other. Baryons with the largest strange quark content, which find themselves at the end of the chain of direct reactions (7) and have the smallest yield before final state interaction, have the largest enhancement. Moreover, the gain in the yield of strange baryons is larger than the one of antibaryons since $\rho_{B}>\rho_{\bar{B}}$. Furthermore, the enhancement of all baryon species increases with centrality, since the gain, resulting from the first term in eq. (6), contains a product of densities and thus, increases quadratically with increasing centrality.

Although the inverse slopes ("temperature") have not been discussed here, let us note that in DPM they are approximately the same for all baryons and antibaryons both before and after final state interaction - the effect of final state interaction being rather small [16] [23].

In conclusion, we would like to emphasize the fact that in DPM (before final state interaction) the rapidity density of charged particle per participant increases with centrality. This increase is larger for low centralities 10. This has an important effect on both the size and the pattern of strangeness enhancement in our results. It explains why the departure from a linear increase of $\Xi$ 's and $\Omega$ 's (concave shape) seen in Figs. 1c and 1d is also more pronounced for lower centralities. It leads to the convexity in the centrality dependence of the yields of hyperons and antihyperons per participant in Figs. 2 (Note, however, a change of curvature for very peripheral collisions where the effect of final state interaction is negligible). This centrality pattern is a distinctive feature as well as a firm prediction of our approach.

\section{Acknowledgments}

It is a pleasure to thank N. Armesto, A. Kaidalov, K. Redlich and Yu. Shabelski for discussions and C. Roy (STAR) and M.J. Tannenbaum (PHENIX) for information on the data. C.A.S. is supported by a Marie Curie Fellowship of the European Community program TMR (Training and Mobility of Researchers), under the contract number HPMF-CT-2000-01025. 


\section{References}

1. WA97 coll., E. A. Andersen et al, Phys. Lett. B433, 209 (1998) ; Phys. Lett. B449, 401 (1999).

NA57 coll., N. Carrer, Nucl. Phys. A698, 118c (2002).

2. NA49 coll., H. Appelshäuser et al, Phys. Lett. B433, 523 (1998) ; Phys. Lett. B444, 523 (1998) ; Eur. Phys. J. C2, 661 (1998).

3. A. Capella and C. A. Salgado, New Journal of Physics 2, 30.1-30.4 (2000) ; Phys. Rev. C60, 054906 (1999).

A. Capella, E. G. Ferreiro and C. A. Salgado, Phys. Lett. B459, 27 (1999) ; Nucl. Phys. A661, 502 (1999).

4. A. Capella, U. Sukhatme, C.-I. Tan and J. Tran Thanh Van, Phys. Lett. 81B, 68 (1979) ; Phys. Rep. 236, 225 (1994).

5. G. C. Rossi and G. Veneziano, Nucl. Phys. B123, 507 (1977).

6. B. Z. Kopeliovich and B. G. Zakharov, Sov. J. Nucl. Phys. 48, 136 (1988) ; Z. Phys. C43, 241 (1989).

7. D. Kharzeev, Phys. Lett. B378, 238 (1996).

8. A. Capella and B. Z. Kopeliovich, Phys. Lett. B381, 325 (1996).

9. S. E. Vance and M. Gyulassy, Phys. Rev. Lett. 83, 1735 (1999).

10. A. Capella and D. Sousa, Phys. Lett. B511, 185 (2001).

11. G. H. Arakelyan, A. Capella, A. Kaidalov and Yu. M. Shabelski, hep-ph/0103337, to be published in Z. Phys. C.

12. A. B. Kaidalov, Phys. Lett. 116B, 459 (1982).

13. E769 coll., E. M. Aitala et al., Phys. Lett. B496, 9 (2000).

14. B. Koch, U. Heinz and J. Pitsut, Phys. Lett. B243, 149 (1990). 
15. A. Capella, A. B. Kaidalov and D. Sousa, nucl-th/0105021, to be published in Phys. Rev. C.

16. N. S. Amelin, N. Armesto, C. Pajares and D. Sousa, Eur. Phys. J. C 22, 149 (2001).

17. PHENIX coll., K. Adcox et al, nucl-ex/0112006.

18. STAR coll., C. Roy, nucl-ex/111017.

19. STAR coll., C. Adler et al, Phys. Rev. Lett. 87, 262302 (2001).

20. STAR coll., H. Caines, Nucl. Phys. A698, 112c (2002).

21. STAR coll., C. Adler et al, Phys. Rev. Lett. 86, 4778 (2001).

22. STAR coll., J. Castillo, Proc. Strangeness in Quark Mater 2001, Frankfurt (Germany) to be published.

23. J. Ranft, A. Capella and J. Tran Thanh Van, Phys. Lett. B320, 346 (1994).

24. PHENIX coll., K.Adcox et al, nucl-ex/0204007.

\section{Figure Caption :}

Figure 1. (a) Calculated values of rapidity densities of $p$ (solid line), $\bar{p}$ (dashed line), and $p-\bar{p}$ (dotted line) at mid rapidities, $\left|y^{*}\right|<0.35$, are plotted as a function of $d N^{-} / d \eta$, and compared with PHENIX data 17] ; (b) same for $\Lambda$ and $\bar{\Lambda}$ compared to preliminary STAR data 19$]$; (c) same for $\Xi^{-}$and $\bar{\Xi}^{+}$; (d) same for $\Omega$ and $\bar{\Omega}$.

Figure 2. Calculated values of the ratios $B / n_{\text {part }}$ (a) and $\bar{B} / n_{\text {part }}$ (b), normalized to the same ratio for peripheral collisions $\left(n_{\text {part }}=18\right)$, are plotted as a function of $n_{\text {part }}$. 
Fig. 1
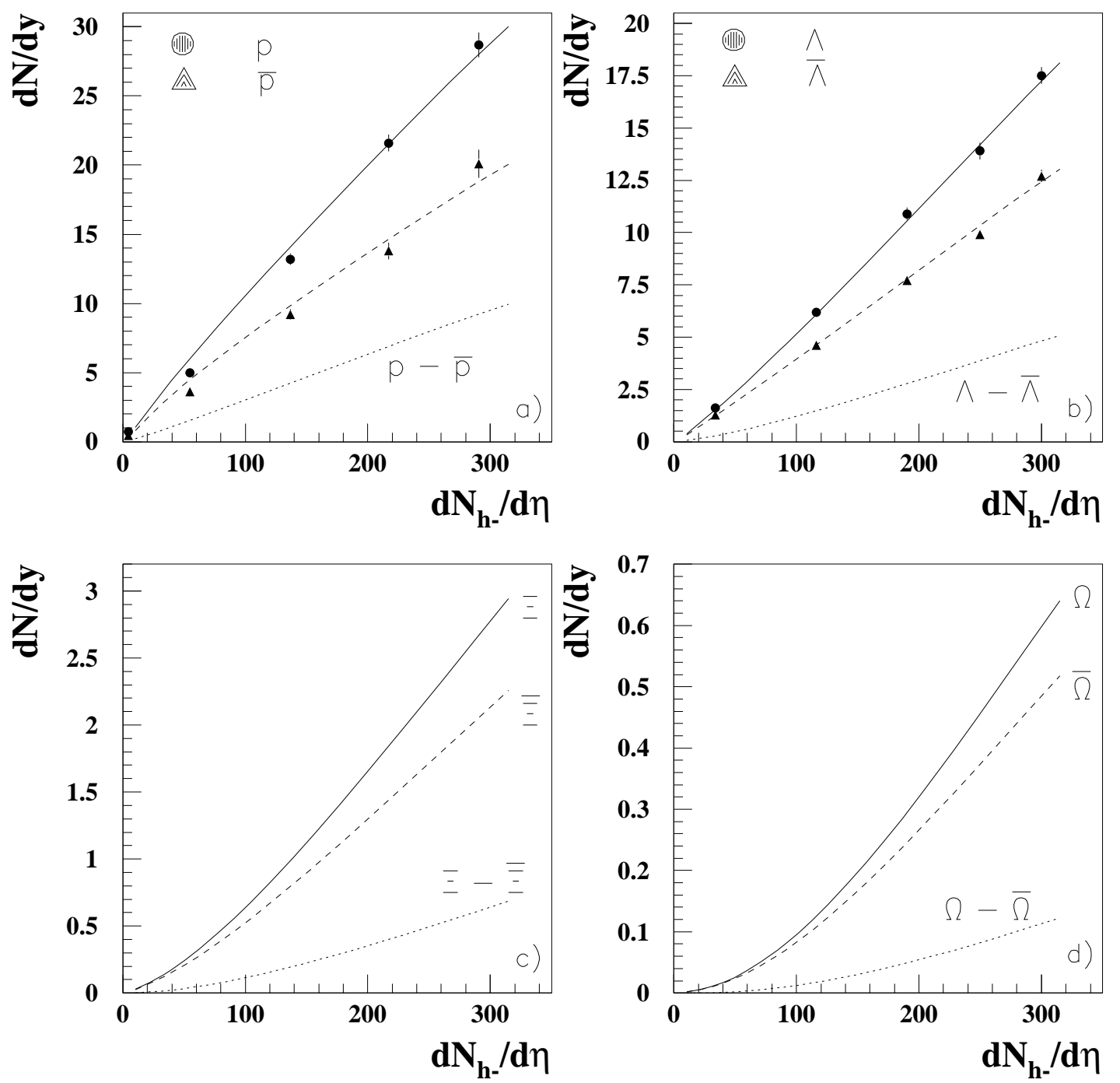
Fig. 2.a

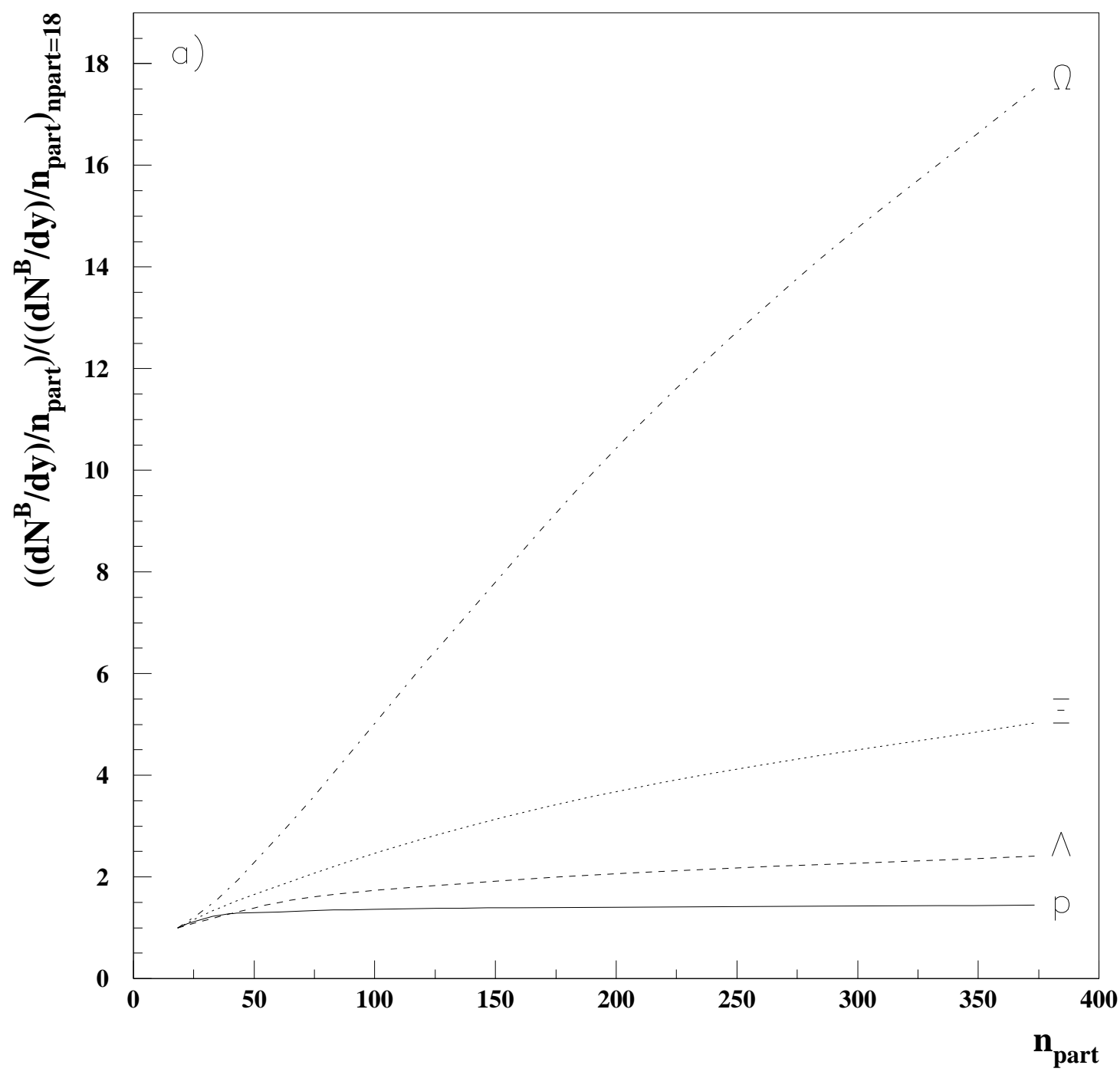


Fig. 2.b

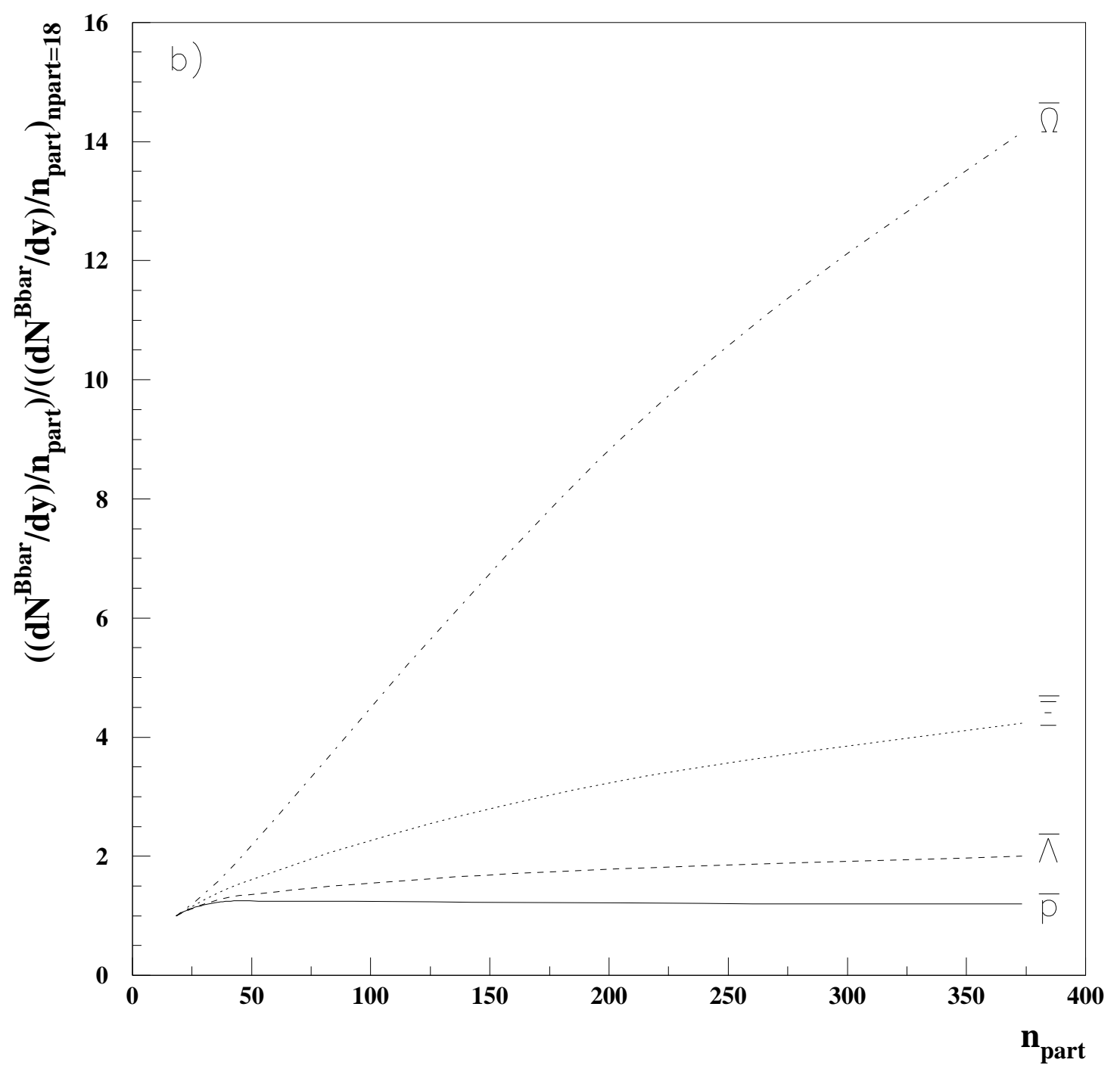




\title{
Strange particle production at RHIC in the Dual Parton Model
}

\author{
A. Capella ${ }^{\text {a) }}$, C.A. Salgado ${ }^{\text {b) }}$, D. Sousa ${ }^{\text {() }}$ \\ a) Laboratoire de Physique Théorique ${ }^{1}$ \\ Université de Paris XI, Bâtiment 210, F-91405 Orsay Cedex, France \\ b) Theory Division, CERN, CH-1211 Geneva 23, Switzerland \\ c) $\mathrm{ECT}^{*}$, Trento, Italy
}

\begin{abstract}
We compute the mid-rapidity densities of pions, kaons, baryons and antibaryons in $A u-A u$ collisions at $\sqrt{s}=130 \mathrm{GeV}$ in the Dual Parton Model supplemented with final state interaction (comovers interaction). The ratios $B / n_{\text {part }}\left(\bar{B} / n_{\text {part }}\right)$ increase between peripheral $\left(n_{\text {part }}=18\right)$ and central $\left(n_{\text {part }}=350\right)$ collisions by a factor $2.4(2.0)$ for $\Lambda$ 's, 4.8 (4.1) for $\Xi$ 's and 16.5 (13.5) for $\Omega^{\prime}$ 's. The ratio $K^{-} / \pi^{-}$increases by a factor 1.3 in the same centrality range. A comparison with available data is presented.
\end{abstract}

LPT Orsay 02-09

CERN-TH/2002-090

$\mathrm{ECT}^{*}$ 02-10

February 2002

\footnotetext{
${ }^{1}$ Unité Mixte de Recherche UMR n 8627 - CNRS
} 
The enhancement of the ratio of yields of strange baryons and antibaryons per participant nucleon, observed at CERN-SPS, [1] [2], is one of the main results of the Heavy Ion CERN program. A description of these data has been given in [3] in the framework of the Dual Parton Model DPM [4], supplemented with final state interaction. The net baryon yield is computed in the same framework taking into account the mechanism of baryon stopping, associated with baryon junction transfer in rapidity [5-9]. We use its implementation in [3], which describes the SPS data.

In the absence of nuclear shadowing, the rapidity density of a given type of hadron $h$ produced in $A A$ collisions at fixed impact parameter, is given by [4] [10]

$$
\begin{aligned}
\frac{d N^{A A \rightarrow h}}{d y}(y, b)= & n_{A}(b)\left[N_{h, \mu(b)}^{q q^{P}-q_{v}^{T}}(y)+N_{h, \mu(b)}^{q_{v}^{P}-q q^{T}}(y)+(2 k-2) N_{h, \mu(b)}^{q_{s}-\bar{q}_{s}}(y)\right] \\
& +\left(n(b)-n_{A}(b)\right) 2 k N_{h, \mu(b)}^{q_{s}-\bar{q}_{s}}(y) .
\end{aligned}
$$

Here

$$
n(b)=\sigma_{p p} A^{2} \int d^{2} s T_{A}(s) T_{B}(b-s) / \sigma_{A A}(b)
$$

is the average number of binary collisions and

$$
n_{A}(b)=A \int d^{2} s T_{A}(s)\left[1-\exp \left(-\sigma_{p p} A T_{A}(b-s)\right] / \sigma_{A A}(b),\right.
$$

is the average number of participant pairs at fixed impact parameter $b . P$ and $T$ denote projectile and target nuclei. $k$ is the average number of inelastic collisions in $p p$ and $\mu(b)=k \nu(b)$ with $\nu(b)=n(b) / n_{A}(b)$ is the average total number of collisions suffered by each nucleon. At $\sqrt{s}=130 \mathrm{GeV}$ we have $k=2$ [10].

The $N_{h, \mu(b)}(y)$ in eq. (1) are the rapidity distributions of hadron $h$ in each individual string. In DPM they are given by convolutions of momentum distribution and fragmentation functions ${ }^{2}$. The first term in (1) is the rapidity distribution in one $N N$ collision of $A A$, resulting from the superposition of $2 k$ strings, multiplied by the average number of participant pairs. Since in DPM there are two strings

\footnotetext{
${ }^{2}$ For pions, we use the same fragmentation functions given in [10]. For simplicity, the same form is used for kaons. For $p \bar{p}$ pair production we take [11] $x D_{q q}^{p}(x)=x D_{q q}^{\bar{p}}(x) \sim(1-x)^{5}$ and $x D_{q}^{p}(x)=x D_{q}^{\bar{p}}(x) \sim(1-x)^{3}$. For the other baryon species an extra $\alpha_{\rho}(0)-\alpha_{\phi}(0)=1 / 2$ is added to the power of $(1-x)$ for each strange quark in the baryon [11].
} 
per inelastic collision, the second term, consisting of strings stretched between sea quarks and antiquarks, makes up for the total average number of strings $2 k n(b)$.

It was shown in [10] that eq. (1), supplemented with shadowing corrections, leads to values of charged multiplicities at mid-rapidities as a function of centrality in agreement with data, both at SPS and RHIC. Here we use the same shadowing corrections as in ref. [10] - leading to the lower edge of the shaded area in Fig. 4 of $[10]$.

Net Baryon Production. Let us now consider the net baryon production $\Delta B=$ $B-\bar{B}$. In the standard version of DPM [4] (or QGSM [12]) the leading baryon results from the fragmentation of a valence diquark. This component will be called diquark preserving (DP). The stopping observed in $\mathrm{Pb} P b$ collisions at SPS has led to the introduction of a new mechanism based on the transfer in rapidity of the baryon junction [5-9]. Here we follow the formalism in [3] which describes the SPS data. In an $A A$ collision, this component, called diquark-breaking (DB), gives the following rapidity distribution of the two net baryons in a single $N N$ collision of $A A$ $[3]$

$$
\left(\frac{d N_{D B}^{\Delta B}}{d y}(y)\right)_{\nu(b)}=C_{\nu(b)}\left[Z_{+}^{1 / 2}\left(1-Z_{+}\right)^{\nu(b)-3 / 2}+Z_{-}^{1 / 2}\left(1-Z_{-}\right)^{\nu(b)-3 / 2}\right]
$$

where $Z_{ \pm}=\exp \left( \pm y-y_{\max }\right)$ and $\nu(b)=n(b) / n_{A}(b) . C_{\nu(b)}$ is determined from the normalization to two at each $b$.

The net baryon rapidity distribution in $A A$ collisions is then given by

$$
\frac{d N^{A A \rightarrow \Delta B}}{d y}(y, b)=n_{A}(b)\left[\frac{1}{\nu(b)}\left(\frac{d N_{D P}^{\Delta B}}{d y}(y)\right)_{\nu(b)}+\frac{\nu(b)-1}{\nu(b)}\left(\frac{d N_{D B}^{\Delta B}}{d y}(y)\right)_{\nu(b)}\right]
$$

The physical content of eq. (5) is as follows. Each nucleon interacts in average with $\nu(b)$ nucleons of the other nucleus. It has been argued in [3] that in only one of these collisions the string junction, carrying the baryon number, follows a valence diquark, which fragments according to the DP mechanism. In the $\nu(b)-1$ others, the string junction is freed from the valence diquark and net baryon production takes place according to the DB mechanism, eq. (4). In order to conserve baryon 
number, we have to divide by $\nu(b)$ and multiply by the number of participating nucleons. We obtain in this way eq. $(5)^{3}$. This equation gives the total net baryon density ${ }^{4}$.

In order to get the relative densities of each baryon and antibaryon species we use simple quark counting rules [3]. Denoting the strangeness suppression factor by $S / L$ (with $2 L+S=1$ ), baryons produced out of three sea quarks (which is the case for pair production) are given the relative weights

$$
I_{3}=4 L^{3}: 4 L^{3}: 12 L^{2} S: 3 L S^{2}: 3 L S^{2}: S^{3} .
$$

for $p, n, \Lambda+\Sigma, \Xi^{0}, \Xi^{-}$and $\Omega$, respectively. The various coefficients of $I_{3}$ are obtained from the power expansion of $(2 L+S)^{3}$. In order to take into account the decay of $\Sigma^{*}(1385)$ into $\Lambda \pi$, we redefine the relative rate of $\Lambda$ 's and $\Sigma$ 's using the empirical rule $\Lambda=0.6\left(\Sigma^{+}+\Sigma^{-}\right)$- keeping, of course, the total yield of $\Lambda^{\prime}$ s plus $\Sigma$ 's unchanged. In this way the normalization constants of all baryon species in pair production are determined from one of them. This constant, together with the relative normalization of $K$ and $\pi$, are determined from the data for very peripheral collisions. In the calculations we use $S=0.1(S / L=0.22)$.

For net baryon production two possibilities have been considered. The first one is that the behaviour in $Z^{1 / 2}$, eq. (4), is associated to the transfer of the string junction without valence quarks $[5,7,9]$. In this case the net baryon is made out of three sea quarks and the relevant weights are given by $I_{3}$. In the second one, eq. (4) is a pre-asymptotic term associated to the transfer of the baryon junction plus one valence quark [6]. In this case the relevant weights are given by $I_{2}$, i.e. from the various terms in the expansion of $(2 L+S)^{2}$. This second possibility is favored by the data ${ }^{5}$. Since the normalization of the total net baryon yield is determined

\footnotetext{
${ }^{3}$ In the numerical calculations we neglect the first term of (5) since the DP component gives a very small contribution at $y^{*} \sim 0$ and RHIC energies - about $5 \%$ of the $D B$ one for the most central bin where its effect is maximal.

${ }^{4}$ In order to conserve strangeness locally, we have added an extra $1 / 2 K^{+}$and $1 / 2 K^{0}$ to each produced net $\Lambda$ (plus $\Sigma$ 's), an extra $K^{+}$and $K^{0}$ to each net $\Xi$ and an extra $3 / 2 K^{+}$and $3 / 2 K^{0}$ to each net $\Omega$.

${ }^{5}$ Note, however, that a non-zero value of net omegas has been observed in $h A$ collisions [13]. This requires a non-vanishing contribution proportional to $I_{3}$. However, its effect in $A A$ collisions is presumably small since, in this case, the net omegas are almost entirely due to final state
} 
from baryon number conservation, there is no extra free normalization constant. Moreover, the total net baryon yield is not affected by final state interaction.

Final State Interactions. The hadronic densities obtained above will be used as initial conditions in the gain and loss differential equations which govern final state interactions. In the conventional derivation [14] of these equations, one uses cylindrical space-time variables and assumes boost invariance. Furthermore, one assumes that the dilution in time of the densities is only due to longitudinal motion, which leads to a $\tau^{-1}$ dependence on the longitudinal proper time $\tau$. These equations can be written as [14] [3]

$$
\tau \frac{d \rho_{i}}{d \tau}=\sum_{k \ell} \sigma_{k \ell} \rho_{k} \rho_{\ell}-\sum_{k} \sigma_{i k} \rho_{i} \rho_{k} .
$$

The first term in the r.h.s. of (6) describes the production (gain) of particles of type $i$ resulting from the interaction of particles $k$ and $\ell$. The second term describes the loss of particles of type $i$ due to its interaction with particles of type $k$. In eq. (6) $\rho_{i}=d N_{i} / d y d^{2} s(y, b)$ are the particles yields per unit rapidity and per unit of transverse area, at fixed impact parameter. They can be obtained from the rapidity densities (1), (5) using the geometry, i.e. the $s$-dependence of $n_{A}$ and $n$, eqs. (2), (3). The procedure is explained in detail in [15]. $\sigma_{k \ell}$ are the corresponding cross-sections averaged over the momentum distribution of the colliding particles.

Equations (6) have to be integrated from initial time $\tau_{0}$ to freeze-out time $\tau_{f}$. They are invariant under the change $\tau \rightarrow c \tau$ and, thus, the result depends only on the ratio $\tau_{f} / \tau_{0}$. We use the inverse proportionality between proper time and densities and put $\tau_{f} / \tau_{0}=\left(d N / d y d^{2} s(y, b)\right) / \rho_{f}$. Here the numerator is given by the DPM particles densities. We take $\rho_{f}=\left[3 / \pi R_{p}^{2}\right]\left(d N^{-} / d y\right)_{y^{*} \sim 0}=2 \mathrm{fm}^{-2}$, which corresponds to the charged density per unit rapidity in a $p p$ collision at $\sqrt{s}=$ $130 \mathrm{GeV}$. This density is about $70 \%$ larger [10] than at SPS energies. Since the corresponding increase in the $A A$ density is comparable, the average duration time of the interaction will be approximately the same at CERN SPS and RHIC - about 5 to $7 \mathrm{fm}$.

interactions. 
Next, we specify the channels that have been taken into account in our calculations. They are

$$
\pi N \rightleftarrows K \Lambda(\Sigma), \quad \pi \Lambda(\Sigma) \rightleftarrows K \Xi, \quad \pi \Xi \rightleftarrows K \Omega
$$

We have also taken into account the strangeness exchange reactions

$$
\pi \Lambda(\Sigma) \rightleftarrows K N, \quad \pi \Xi \rightleftarrows K \Lambda(\Sigma), \quad \pi \Omega \rightleftarrows K \Xi
$$

as well as the channels corresponding to (7) and (8) for antiparticles ${ }^{6}$. We have taken $\sigma_{i k}=\sigma=0.2 \mathrm{mb}$, i.e. a single value for all reactions in (7) and (8) - the same value used in ref. [3] to describe the CERN SPS data.

Numerical Results. All our results refer to mid-rapidities. The calculations have been performed in the interval $-0.35<y^{*}<0.35$. In Fig. 1a-1d we show the rapidity densities of $B, \bar{B}$ and $B-\bar{B}^{7}$ versus $h^{-}=d N^{-} / d \eta=(1 / 1.17) d N / d y$ and compare them with available data [17-19]. We see that, in first approximation, $p$, $\bar{p}, \Lambda$ and $\bar{\Lambda}$ scale with $h^{-}$. Quantitatively, there is a slight decrease with centrality of $p / h^{-}$and $\bar{p} / h^{-}$ratios, a slight increase of $\Lambda / h^{-}$and $\bar{\Lambda} / h^{-}$and a much larger increase for $\Xi(\bar{\Xi}) / h^{-}$and $\Omega(\bar{\Omega}) / h^{-}$. In Fig. 2a and $2 \mathrm{~b}$ we plot the yields of $B$ and $\bar{B}$ per participant normalized to the same ratio for peripheral collisions versus $n_{\text {part }}$. The enhancement of $B$ and $\bar{B}$ increases with the number of strange quarks in the baryon. This increase is comparable to the one found at SPS between pA and central $\mathrm{PbPb}$ collisions - somewhat larger for antibaryons. The ratio $K^{-} / \pi^{-}$ increases by $30 \%$ in the same centrality range, between 1.1 and 1.4 in agreement with present data [17]. The ratios $\bar{B} / B$ have a mild decrease with centrality of

\footnotetext{
${ }^{6}$ To be precise, of all possible charge combinations in reactions (7), we have only kept those involving the annihilation of a light $q-\bar{q}$ pair and production of an $s-\bar{s}$ in the $s$-channel. The other reactions, involving three quarks in the $t$-channel intermediate state, have substantially smaller cross-sections and have been neglected. All channels involving $\pi^{0}$ have been taken with crosssection $\sigma / 2$ since only one of the $u \bar{u}$ and $d \bar{d}$ components of $\pi^{0}$ can participate to a given charge combination. For details see the second paper of [3].

${ }^{7} \mathrm{~A}$ Monte Carlo calculation in a similar framework with string fusion can be found in [16]. A net proton rapidity density of about 10 for central $A u A u$ collisions at mid-rapidities at RHIC was first predicted in [8] using a stopping mechanism similar to the one considered here.
} 
about $15 \%$ for all baryon species - which is also seen in the data [21]. Our values for $N^{c h} / N_{\max }^{c h}=1 / 2$ are $: \bar{p} / p=0.69, \bar{\Lambda} / \Lambda=0.72, \bar{\Xi} / \Xi=0.79, \Omega / \bar{\Omega}=0.83^{8}$, to be compared with the measured values [18] :

$$
\bar{p} / p=0.63 \pm 0.02 \pm 0.06 \quad, \quad \bar{\Lambda} / \Lambda=0.73 \pm 0.03 \quad, \quad \bar{\Xi} / \Xi=0.83 \pm 0.03 \pm 0.05
$$

The ratio $K^{+} / K^{-}=1.1$ and has a mild increase with centrality, a feature also seen in the data.

As explained above only one parameter has been adjusted in order to determine the absolute yields of baryons and antibaryons. Since both the PHENIX [17] and the STAR data [19] are not corrected for feed-down from weak decays, this free parameter has to be re-determined after these corrections are known. It will then be possible to compare its value with the one obtained from other sets of data, in particular $p p$. Likewise, the corrected yields of net protons will determine the exact amount of stopping and should allow to decide whether or not there is "anomalous" stopping in $A A$, i.e. an excess as compared to an extrapolation from $p p$ and $p A$. At present there is no clear indication of anomalous stopping.

After completion of this work, the PHENIX collaboration [24] has published the yields of $p$ and $\bar{p}$ for the $5 \%$ most central events corrected for feed-down. The corrections are of $30 \%$. The systematic error is $20 \%$.Also, C.Roy (STAR collaboration) communicated to us a recent STAR preliminary result on the mid-rapidity densities of $\Xi^{-}$and $\Xi^{+}$for the $14 \%$ most central events. These values are 60 to $70 \%$ higher than our results. Their systematic error is $20 \%$.

Physical interpretation. Before final state interactions, all ratios $K / h^{-}, B / h^{-}$ and $\bar{B} / h^{-}$decrease slightly with increasing centrality. This effect is rather marginal at RHIC energies and mid-rapidities.

The final state interactions (7), (8) lead to a gain of strange particle yields. The reason for this is the following. In the first direct reaction (7) we have $\rho_{\pi}>\rho_{K}$, $\rho_{N}>\rho_{\Lambda}, \rho_{\pi} \rho_{N} \gg \rho_{K} \rho_{\Lambda}$. The same is true for all direct reaction (7). In view of that, the effect of the inverse reactions (7) is small. On the contrary, in all reactions

\footnotetext{
${ }^{8}$ In ref. [3], the relative weights of net baryons were given by the factors $0.5\left(I_{2}+I_{3}\right)$ - instead of $I_{2}$. In this case the values of the ratios are $\bar{p} / p=0.70, \bar{\Lambda} / \Lambda=0.71, \bar{\Xi} / \Xi=0.76$ and $\bar{\Omega} / \Omega=0.78$. Their increase with the number of strange quarks in the baryon is smaller.
} 
(8), the product of densities in the initial and final state are comparable and the direct and inverse reactions tend to compensate with each other. Baryons with the largest strange quark content, which find themselves at the end of the chain of direct reactions (7) and have the smallest yield before final state interaction, have the largest enhancement. Moreover, the gain in the yield of strange baryons is larger than the one of antibaryons since $\rho_{B}>\rho_{\bar{B}}$. Furthermore, the enhancement of all baryon species increases with centrality, since the gain, resulting from the first term in eq. (6), contains a product of densities and thus, increases quadratically with increasing centrality.

Although the inverse slopes ("temperature") have not been discussed here, let us note that in DPM they are approximately the same for all baryons and antibaryons both before and after final state interaction - the effect of final state interaction being rather small [16] [23].

In conclusion, we would like to emphasize the fact that in DPM (before final state interaction) the rapidity density of charged particle per participant increases with centrality. This increase is larger for low centralities [10]. This has an important effect on both the size and the pattern of strangeness enhancement in our results. It explains why the departure from a linear increase of $\Xi$ 's and $\Omega$ 's (concave shape) seen in Figs. 1c and 1d is also more pronounced for lower centralities. It leads to the convexity in the centrality dependence of the yields of hyperons and antihyperons per participant in Figs. 2 (Note, however, a change of curvature for very peripheral collisions where the effect of final state interaction is negligible). This centrality pattern is a distinctive feature as well as a firm prediction of our approach.

\section{Acknowledgments}

It is a pleasure to thank N. Armesto, A. Kaidalov, K. Redlich and Yu. Shabelski for discussions and C. Roy (STAR) and M.J. Tannenbaum (PHENIX) for information on the data. C.A.S. is supported by a Marie Curie Fellowship of the European Community program TMR (Training and Mobility of Researchers), under the contract number HPMF-CT-2000-01025. 


\section{References}

1. WA97 coll., E. A. Andersen et al, Phys. Lett. B433, 209 (1998) ; Phys. Lett. B449, 401 (1999).

NA57 coll., N. Carrer, Nucl. Phys. A698, 118c (2002).

2. NA49 coll., H. Appelshäuser et al, Phys. Lett. B433, 523 (1998) ; Phys. Lett. B444, 523 (1998) ; Eur. Phys. J. C2, 661 (1998).

3. A. Capella and C. A. Salgado, New Journal of Physics 2, 30.1-30.4 (2000) ; Phys. Rev. C60, 054906 (1999).

A. Capella, E. G. Ferreiro and C. A. Salgado, Phys. Lett. B459, 27 (1999) ; Nucl. Phys. A661, 502 (1999).

4. A. Capella, U. Sukhatme, C.-I. Tan and J. Tran Thanh Van, Phys. Lett. 81B, 68 (1979) ; Phys. Rep. 236, 225 (1994).

5. G. C. Rossi and G. Veneziano, Nucl. Phys. B123, 507 (1977).

6. B. Z. Kopeliovich and B. G. Zakharov, Sov. J. Nucl. Phys. 48, 136 (1988) ; Z. Phys. C43, 241 (1989).

7. D. Kharzeev, Phys. Lett. B378, 238 (1996).

8. A. Capella and B. Z. Kopeliovich, Phys. Lett. B381, 325 (1996).

9. S. E. Vance and M. Gyulassy, Phys. Rev. Lett. 83, 1735 (1999).

10. A. Capella and D. Sousa, Phys. Lett. B511, 185 (2001).

11. G. H. Arakelyan, A. Capella, A. Kaidalov and Yu. M. Shabelski, hep-ph/0103337, to be published in Z. Phys. C.

12. A. B. Kaidalov, Phys. Lett. 116B, 459 (1982).

13. E769 coll., E. M. Aitala et al., Phys. Lett. B496, 9 (2000).

14. B. Koch, U. Heinz and J. Pitsut, Phys. Lett. B243, 149 (1990). 
15. A. Capella, A. B. Kaidalov and D. Sousa, nucl-th/0105021, to be published in Phys. Rev. C.

16. N. S. Amelin, N. Armesto, C. Pajares and D. Sousa, Eur. Phys. J. C 22, 149 (2001).

17. PHENIX coll., K. Adcox et al, nucl-ex/0112006.

18. STAR coll., C. Roy, nucl-ex/111017.

19. STAR coll., C. Adler et al, Phys. Rev. Lett. 87, 262302 (2001).

20. STAR coll., H. Caines, Nucl. Phys. A698, 112c (2002).

21. STAR coll., C. Adler et al, Phys. Rev. Lett. 86, 4778 (2001).

22. STAR coll., J. Castillo, Proc. Strangeness in Quark Mater 2001, Frankfurt (Germany) to be published.

23. J. Ranft, A. Capella and J. Tran Thanh Van, Phys. Lett. B320, 346 (1994).

24. PHENIX coll., K.Adcox et al, nucl-ex/0204007.

\section{Figure Caption :}

Figure 1. (a) Calculated values of rapidity densities of $p$ (solid line), $\bar{p}$ (dashed line), and $p-\bar{p}$ (dotted line) at mid rapidities, $\left|y^{*}\right|<0.35$, are plotted as a function of $d N^{-} / d \eta$, and compared with PHENIX data [17] ; (b) same for $\Lambda$ and $\bar{\Lambda}$ compared to preliminary STAR data [19]; (c) same for $\Xi^{-}$and $\bar{\Xi}^{+}$; (d) same for $\Omega$ and $\bar{\Omega}$.

Figure 2. Calculated values of the ratios $B / n_{\text {part }}$ (a) and $\bar{B} / n_{\text {part }}$ (b), normalized to the same ratio for peripheral collisions $\left(n_{\text {part }}=18\right)$, are plotted as a function of $n_{\text {part }}$. 
Fig. 1
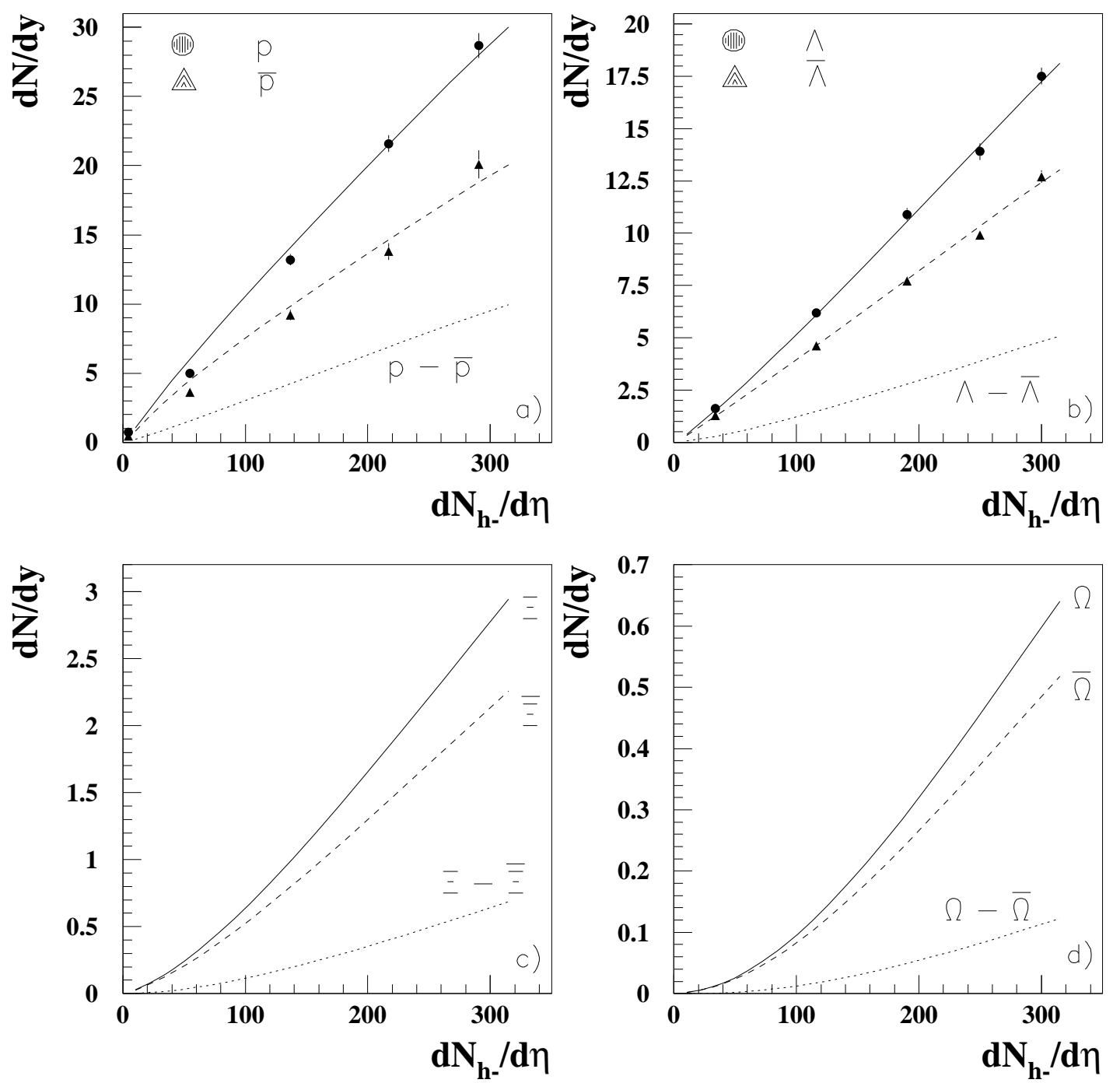
Fig. 2.a

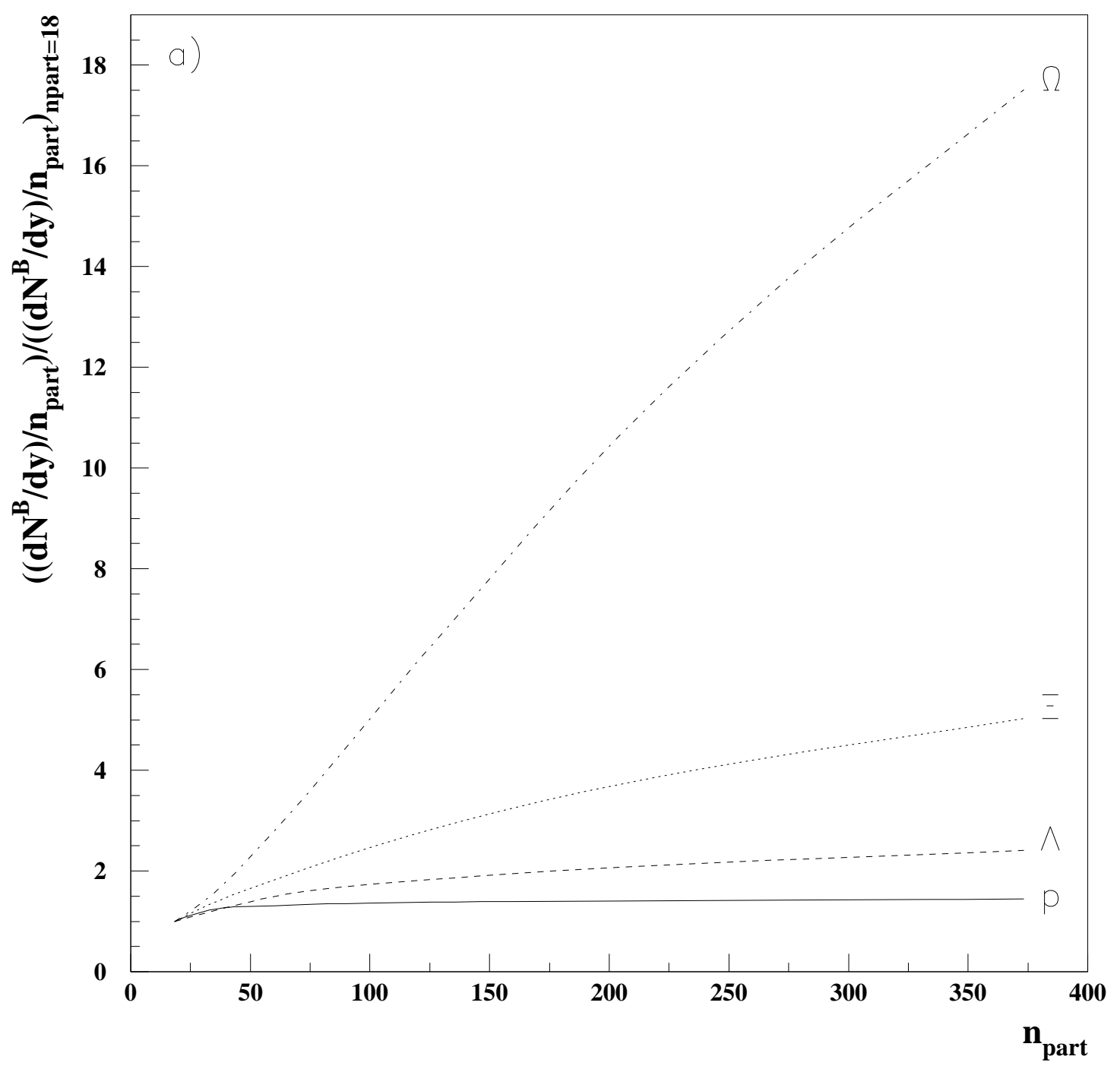


Fig. 2.b

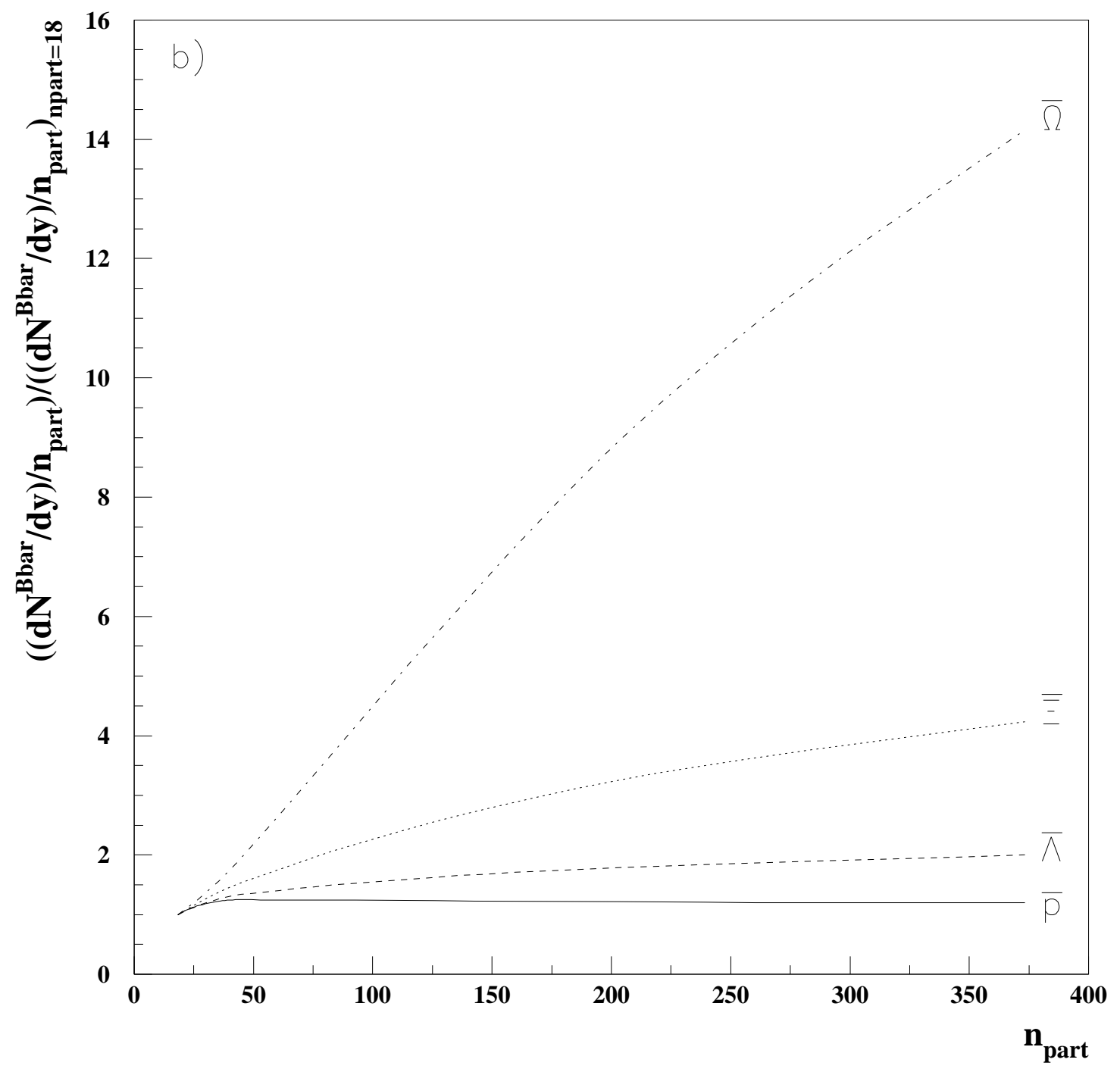

